Ann. Génét. Sél. anim., 1975, 7 (3), 293-298.

\title{
SIMPLIFICATION DE L'ÉCHANTILLONNAGE EN VUE DU CONTRÔLE LAITIER QUALITATIF DES BREBIS ET DES CHÈVRES ( $\left.{ }^{1}\right)$
}

\author{
R. CARTA, S. CASU, J. C. FLAMANT* et G. RICORDEAU* \\ Istituto Zootecnico e Caseario per la Sardegna, \\ Sassari (Italie) \\ * Laboratoire de Génétique des Petits Ruminants, \\ Centre de Recherches de Toulouse, I. N.R.A., \\ 31320 Castanet Tolosan (France)
}

\section{RÉSUMÉ}

Les résultats d'analyses effectuées systématiquement tous les 7 ou I4 jours à chacune des deux traites journalières sur les laits de brebis Sardes et Lacaune et de chèvres Alpines Chamoisées et Saanen recueillis dans différents troupeaux expérimentaux sont utilisés en vue d'étudier les possibilités de simplification des prélèvements de lait :

- prélèvements égaux à chaque traite,

- prélèvement à l'une des traites journalières,

- prélèvements bimestriels au lieu de mensuels.

Il apparaît que pour les deux espèces ces simplifications sont assez difficilement envisageables pour l'estimation de la teneur en matière grasse. Par contre, la réalisation de prélèvements égaux le matin et le soir, ou des contrôles bimestriels sont possibles pour estimer la teneur en matière azotée sans perte appréciable de précision ni erreur systématique importante par rapport à la méthode classique de prélèvement mensuel proportionnel (moins de $\mathrm{x}$ p. roo) ; éventuellement, on peut envisager aussi un seul prélèvement journalier qui devrait porter dans ce cas sur le lait de la traite du matin.

\section{INTRODUCTION}

La mise au point de méthodes colorimétriques pour le dosage de la matière azotée, et 1'utilisation d'appareils automatiques, ont rendu plus facile l'estimation de la teneur des laits. Cependant, le coût relatif des analyses des laits de brebis et

( $\left.{ }^{1}\right)$ Communication au Symposium international sur le contrôle laitier des brebis et des chèvres. Israël, 19-24 mars 1972 . 
de chèvre reste élevé compte tenu du faible volume des productions de ces animaux. La prise en compte des critères fromagers dans les schémas de sélection de ces 2 espèces est en conséquence limitée. Aussi avons-nous envisagé différentes possibilités de simplification des manipulations nécessaires à la constitution de chaque échantillon d'analyse (prélèvement à l'une des traites journalières ou prélèvements égaux à chacune des traites du soir et du matin), ainsi que la diminution du nombre d'échantillons par lactation (prélèvements bimestriels au lieu de mensuels).

\section{MATÉRIEL, ET MÉTHODES}

Les données ont été recueillies dans des troupeaux expérimentaux ovins ou caprins appartenant à I'Istituto Zootecnico e Caseario per la Sardegna (brebis Sardes, à Bonassai) ou à l'Institut National de la Recherche Agronomique (brebis Sardes et Lacaune, à La Fage; chèvres Alpines Chamoisées et Saanen, à Galle).

Les teneurs en matière grasse ont été estimées par la méthode Gerber. Pour la matière azotée on a utilisé à Bonassai la méthode colorimétrique à l'orange G, à La Fage un appareil automatique Technicon étalonné par la méthode colorimétrique au Noir Amido, et à Galle la méthode au Noir Amido mise au point spécialement pour les laits de chèvres par la Station I. N. R. A. de Recherches laitières de Poligny (Jura).

\section{I. - Simplification des prélèvements journaliers}

Les prélèvements ont été effectués à chacune des 2 traites journalières :

- à Bonassai, tous les 7 jours, sur 30 brebis Sardes durant 25 semaines (intervalle des traites matin-soir ; 12 heures),

- à La Fage, tous les $\mathrm{r}_{4}$ jours, sur 60 brebis Sardes et 80 brebis Lacaune, durant $\mathrm{I} 8$ semaines (intervalle des traites matin-soir; Io heures),

- à Galle, tous les 14 jours, sur 76 chèvres avec un intervalle de traites matin-soir de 12 heures durant un mois et sur 87 chèvres avec un intervalle de ro heures durant 4 mois.

Pour chaque animal $i$ et pour chaque jour de contrôle $j$, on a estimé la teneur journalière à partir des teneurs observées à chacune des 2 traites :

- moyenne arithmétique $\left(\mathrm{A}_{i j}\right)$, qui simule la réalisation des prélèvements égaux à chacune des 2 traites quotidiennes (méthode A),

-- teneurs à la traite du matin $\left(\mathrm{M}_{i j}\right)$ ou du soir $\left(\mathrm{S}_{i j}\right)$ qui correspondent à un seul prélèvement par jour (méthodes $M$ et $S$ ).

La moyenne arithmétique pondérée des teneurs $\left(\mathrm{P}_{i j}\right)$, tenant compte des quantités de lait de chacune des 2 traites, est considérée comme étant l'estimation la plus précise et simule la méthode des prélèvements proportionnels habituellement employée (méthode P).

Pour chaque jour de contrôle et pour l'ensemble des animaux, chacune des méthodes étudiées $(\mathrm{A}, \mathrm{M}$ et $\mathrm{S}$ ) est mise en comparaison avec la méthode des prélèvements proportionnels $(\mathrm{P})$ à l'aide des paramètres suivants :

- teneur estimée par l'une des méthodes $\mathrm{A}, \mathrm{M}$ ou $\mathrm{S}: \mathrm{E}_{i j}$

- moyenne et variance des teneurs estimées par la méthode $\mathrm{P}: \overline{p_{j}}$ et $s_{\boldsymbol{p}_{j}}^{2}$

et, si $d_{i j}=\left(\mathrm{E}_{i j}-\mathrm{P}_{i j}\right)$ représente l'écart entre les estimées individuelles :

- écart moyen $\mathrm{D}: \bar{d}_{j}=\frac{\Sigma_{i} d_{i j}}{n}$

- variance des écarts $\mathrm{S}^{2}: s_{d s}^{2}=\frac{\Sigma_{i}\left(d_{i j}-\bar{d}_{j}\right)^{2}}{n-\mathrm{I}}$

- estimées des erreurs relatives : $e_{d}=\frac{\overline{d_{j}}}{\overline{p_{j}}}$

$$
e_{z_{d}^{2}}^{2}=\frac{s_{d,}^{2}}{s_{p_{1}}^{2}}
$$




\section{2. - Réduction de la périodicité des contrôles}

La teneur moyenne pondérée du lait de r68 lactations de brebis Sardes du troupeau de Bonassai a été estimée à partir de contrôles mensuels $(\mathrm{m})$ ou bimestriels $(\mathrm{Bm})$. La comparaison entre les 2 estimées a été réalisée par le calcul de la différence $d$ entre les 2 estimations (Bm -.. $\mathrm{m}$ ) pour chaque lactation et la détermination de $\overline{\mathrm{D}}$ et $s_{\boldsymbol{d}}$ (moyenne et écart-type des différences).

\section{RÉSULTATS}

Le tableau I rapporte les valeurs moyennes des 4 paramètres, calculées sur l'ensemble des contrôles réalisés : $\overline{\mathrm{D}}, \overline{\mathrm{S}^{2}}, \overline{e_{d}}, \overline{e_{8_{d}^{2}}}$

\section{- DISCUSSION}

Les résultats obtenus sur les diverses espèces ou races en vue de la simplification du contrôle journalier concordent très bien entre eux et peuvent être résumés de la façon suivante :

- l'écart systématique et la variation aléatoire sont toujours plus importants en valeur absolue $\left(\overline{\mathrm{D}}\right.$ et $\left.\overline{\mathrm{S}^{2}}\right)$ et relative $\left(\overline{e_{d}}\right.$ et $\left.\overline{e_{s_{d}^{2}}}\right)$ pour la teneur en matière grasse que pour la matière azotée, et lorsque l'intervalle entre la traite du matin et la traite du soir est plus faible (ro ou II heures) ;

- par rapport à la moyenne pondérée $(\mathrm{P})$, la moyenne arithmétique $(\mathrm{A})$ donne les erreurs les plus faibles;

- les erreurs les plus importantes sont observées avec les estimations effectuées sur les laits de la traite du soir qui constituent la portion la plus faible et la moins riche du lait journalier ;

- pour les estimations effectuées à partir des laits de la traite du matin, les erreurs systématiques (toujours par défaut) sont dans l'ensemble très faibles et n'excèdent pas un gramme par kilo. dans le cas le plus défavorable. La variation aléatoire est approximativement du même ordre de grandeur. Cependant, pour le taux azoté, la variance générale des observations étant également faible, la perte de précision générale peut être relativement importante ( $\mathrm{I}_{3}$ à $\mathrm{I}_{5} \mathrm{p}$. IOo) alors qu'elle est toujours négligeable $(0,2$ à $0,3 \mathrm{p}$. Ioo) lorsqu'on réalise la moyenne arithmétique des teneurs des laits du soir et du matin.

La réalisation de contrôles bimestriels pour la matière grasse et la matière azotée entraîne quant à elle des erreurs toujours très faibles par rapport à des teneurs estimées à partir de contrôles mensuels. Comme dans les cas précédents celles-ci sont plus importantes sur les teneurs en matière grasse (erreur absolue $=+I, 7 \mathrm{I}$ g/kg erreur relative $=2,47 \mathrm{p}$. Ioo). 
a) Comparaison des teneurs moyennes estimées par différentes méthodes de contrôle simplifié (A, M, S), par rapport à la méthode des prélèvements proportionnels:

$\overline{\mathrm{D}}=$ écart moyen absolu

$\overline{\mathrm{S}^{2}}=$ moyenne de la variance des écarts

$\overline{e_{d}}=$ écart moyen relatif (p. Ioo)

$\overline{e_{s a}^{2}}=$ moyenne de la variance des écarts, rapportée a la variance moyenne des teneurs (p. Ioo).

\begin{tabular}{|c|c|c|c|c|c|c|c|c|}
\hline \multirow[t]{2}{*}{$\begin{array}{c}\text { Composant } \\
\text { du lait }\end{array}$} & \multirow[t]{2}{*}{ Espèces } & \multirow[t]{2}{*}{$\begin{array}{c}\text { Origine } \\
\text { des données } \\
\text { et race }\end{array}$} & \multirow{2}{*}{$\begin{array}{c}\text { Intervalle- } \\
\text { traites } \\
\text { matin-soir } \\
\text { (heures) }\end{array}$} & \multirow[t]{2}{*}{$\begin{array}{c}\text { Nombre } \\
\text { de jours } \\
\text { contrôlés }\end{array}$} & \multicolumn{4}{|c|}{$\begin{array}{l}\text { Prélèvements égaux } \\
\text { soir et matin (A) }\end{array}$} \\
\hline & & & & & $\overline{\mathrm{D}}$ & $\overline{e_{a}}$ & $\overline{\mathrm{S}^{2}}$ & $\overline{e_{s}^{8}}$ \\
\hline \multirow{4}{*}{$\begin{array}{l}\text { Matière } \\
\text { grasse }\end{array}$} & \multirow{3}{*}{ Brebis } & $\begin{array}{c}\text { Bonassai } \\
\text { (race Sarde) }\end{array}$ & 12 & 750 & 0,29 & 0,41 & 0,14 & 0,15 \\
\hline & & $\begin{array}{c}\text { La Fage } \\
\text { (race Sarde) }\end{array}$ & 10 & 519 & 2,22 & 3,50 & 1,68 & 1,50 \\
\hline & & $\begin{array}{c}\text { La Fage } \\
\text { (race Lacaune) }\end{array}$ & 10 & 718 & 3,00 & 4,40 & 3,74 & $2,4:$ \\
\hline & Chèvres & Galle & $\begin{array}{l}12 \\
10\end{array}$ & $\begin{array}{l}153 \\
789\end{array}$ & $\begin{array}{l}0,17 \\
0,77\end{array}$ & $\begin{array}{l}0,47 \\
2,52\end{array}$ & $\begin{array}{l}0,06 \\
0,53\end{array}$ & - \\
\hline \multirow{4}{*}{$\begin{array}{l}\text { Matière } \\
\text { azotée }\end{array}$} & \multirow{3}{*}{ Brebis } & $\begin{array}{c}\text { Bonassai } \\
\text { (race Sarde) }\end{array}$ & 12 & 750 & 0,14 & 0,23 & 0,04 & \\
\hline & & $\begin{array}{c}\text { La Fage } \\
\text { (race Sarde) }\end{array}$ & 10 & 519 & 0,20 & 0,36 & 0,09 & 0,30 \\
\hline & & $\begin{array}{c}\text { La Fage } \\
\text { (race Lacaune) }\end{array}$ & 10 & 718 & 0,23 & 0,40 & 0,16 & \\
\hline & Chèvres & Galle & $\begin{array}{l}12 \\
10\end{array}$ & $\begin{array}{l}153 \\
789\end{array}$ & $\begin{array}{r}-0,01 \\
0,05\end{array}$ & $\begin{array}{r}-0,03 \\
0,15\end{array}$ & $\begin{array}{l}0,02 \\
0,03\end{array}$ & - \\
\hline
\end{tabular}


b) Comparaison des teneurs moyennes par lactation estimées par contrôles bimestriels $(\mathrm{Bm})$ par rapport a un controle mensuel:

$$
\begin{aligned}
\overline{\mathrm{D}} & =\text { écart moyen absolu } \\
\overline{e_{d}} & =\text { écart moyen relatif (p. Ioo) } \\
s_{d} & =\text { écart-type des écarts. }
\end{aligned}
$$

\begin{tabular}{|c|c|c|c|c|c|c|c|c|c|c|}
\hline \multicolumn{4}{|c|}{ Matin (M) } & \multicolumn{4}{|c|}{ Soir (S) } & \multicolumn{3}{|c|}{ Bimestriels (Bm) } \\
\hline$\overline{\mathrm{D}}$ & $\overline{e_{d}}$ & $\overline{\mathrm{S}^{2}}$ & $\overline{e_{8 d}^{2}}$ & $\overline{\mathrm{D}}$ & $\overline{e_{d}}$ & $\overline{\mathrm{S}^{2}}$ & $\overline{e_{8 I}}$ & $\overline{\mathrm{D}}$ & $\overline{\mathfrak{e}_{d}}$ & $\overline{s d}$ \\
\hline$-5,10$ & $-7,35$ & 10,30 & 17,29 & 5,50 & 8,00 & 11,30 & 19,04 & 1,71 & 2,47 & $0,3^{\prime}$ ' \\
\hline- & - & 一 & - & - & - & 一 & - & - & - & - \\
\hline - & - & - & - & 一 & - & - & - & - & - & - \\
\hline$-2,09$ & $-5,80$ & 2,82 & - & 2,43 & 6,75 & 3,53 & - & - & - & - \\
\hline$-3,56$ & $-9,89$ & 3,92 & 一 & 5,31 & 14,75 & 8,18 & - & - & - & 一 \\
\hline$-1,00$ & $-1,64$ & 2,70 & 12,85 & 1,10 & 1,84 & 3,10 & 14,76 & $-0,06$ & 0,10 & 0,13 \\
\hline 一 & - & - & - & - & $\div$ & - & - & - & - & - \\
\hline- & - & - & 一 & - & - & - & - & - & - & - \\
\hline$-0,03$ & $-0,09$ & $0,9^{\prime} t$ & - & 0,08 & 0,25 & 1,30 & - & - & - & 一 \\
\hline$-0,16$ & $-0,50$ & 0,25 & - & 0,31 & 0,97 & 0,60 & - & - & 一 & - \\
\hline
\end{tabular}

Méthode d'estimation 


\title{
CONCLUSION
}

Une simplification notable des prises d'échantillons en vue d'estimer les teneurs des laits de brebis et de chèvre peut être réalisée par des prélèvements bimestriels au lieu de mensuels, et en effectuant des prélèvements égaux à chacune des traites. Par sa faible variabilité soir-matin, la teneur matière azotée peut être estimée dans ces conditions avec beaucoup moins de perte de précision que la teneur en matière grasse. Il apparaît même possible de n'estimer la teneur en matière azotée que sur l'une des 2 traites, de préférence celle du matin. Les corrélations génétiques entre la teneur en matière grasse et la teneur en matière azotée étant par ailleurs élevées, ces résultats constituent des arguments supplémentaires pour retenir en priorité la teneur en matière azotée dans les programmes de sélection ovins et caprins sur la qualité fromagère des laits.

Reçu pour publication en septembre 1975.

\author{
SUMMARY \\ SIMPLIFICATION OF SAMPLING \\ IN QUALITATIVE MILK CONTROL, ON EWES AND GOATS
}

Analyses of fat or nitrogen are done systematically on the milk of Sardinian and Lacaune ewes and Alpine Chamois and Saanen goats every 7 or $\mathrm{I}_{4}$ days at each of the two daily milkings. The results are used to study means of simplifying milk sampling in selection plans :

- equal sampling at each milking,

- sampling at one of the daily milkings,

- bimestrial instead of monthly sampling.

For the two species, it is more difficult to carry out the simplifications proposed for estimating the amount of fat than for estimation of nitrogen content.

Using simplified procedures, the proportion of nitrogen can be evaluated with little loss of precision or systematic error as compared to the proportional sampling method :

- by taking equal samplings morning and night (loss of precision : 0.20 to 0.35 p. roo; systematic error : 0.30 to $0.40 \mathrm{p}$. 100;

- by bimestrial instead of monthly monitoring, which involves a systematic error of o.Io p. I0O as against 2.5 p. Ioo for fat.

Eventually, it may be possible to take a single sampling from the morning milking to estimate the proportion of nitrogen : low systematic error (about I p. roo), but high loss of exactness (13 p. 100). 